\title{
Optimization of HF- injection at the 2nd harmonic of ECRH on T-10 tokamak in order to obtain high energy content in plasma
}

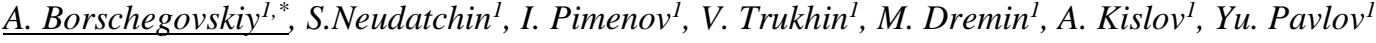 \\ ${ }^{I}$ NRC “Kurchatov Institute”, pl. AkademikaKurchatova 1, Moscow, 123182, Russia
}

\begin{abstract}
Non-traditional scheme of HF-injection has been used in T-10 tokamak for ECRH at the 2nd harmonic of ECR in X-mode. Two HF-launcher systems, with focusing beams, injected power in opposite directions under toroidal angles $+/-20^{\circ}$. Input power from each launcher was about the same $\left(\mathrm{P}_{\mathrm{hf}}=0.8 \div\right.$ $0.85 \mathrm{MWt}$ ). Absorbed energy was deposed in central area of plasma column.

The new phenomenon has been found in some shots with $\mathrm{W}$-limiter and Li-coating in the regime mentioned above. The spontaneous rise of the electron density nearly in the all plasma column occurs simultaneously with the rise of Te in the wide region $(0<\mathrm{r} / \mathrm{a}<0.8)$ at the steady-state of ECRH. The value of the energy confinement time abruptly rises by $\sim 15 \%$. The absolute value of the electron heat and density fluxes reduces abruptly in the whole plasma column similarly to that of at non-local (or global) L-H transitions found earlier in JET and JT-60U. The accumulation of tungsten and light impurities is absent.

In a series of shots, without L-H transitions, measurements of X-ray spectra (PHA data) showed a significant difference in comparison with the ECCD co-injection by two gyrotrons.
\end{abstract}

\section{Introduction}

The main part of experiments on the ECR plasma heating are concentrated on local energy deposition of $\mathrm{HF}$ - power in a rather narrow zone (ECRH perpendicular launching) and generation of noninductive current $(\mathrm{ECCD}$ - oblique launching at toroidal angle $\sim-30^{\circ}$ ).

Non-inductive ECR current (ECCD) is ultimately based on an anisotropic velocity distribution function of electrons in the longitudinal direction of motion. In this case, microwave power is transmitted to the transverse component of the energy of electrons moving in the direction of wave propagation, which obviously leads to a distortion of the electron energy distribution function. Electrons, moving in the opposite direction, do not participate in the ECR heating and their energy distribution function remains Maxwellian due to Coulomb collisions.

Note that the mean value of the kinetic energy of particles in the distribution function, deformed towards higher energies, turns out to be greater than the same value in the Maxwellian distribution (see Fig.1).

$$
\int_{0}^{\infty} f(v) \frac{m v^{2}}{2} d v>\int_{0}^{\infty} f_{\max }(v) \frac{m v^{2}}{2} d v
$$

Therefore in order to increase the electron energy in the ECR area, HF power was injected by two gyrotrons simultaneously in two opposite toroidal directions (co+contr injection). Thus, electrons moving in both directions had resonance with the wave (see Fig.2).

The new scheme of ECRH at the 2nd harmonic in X-mode with simultaneous co-ECCD (toroidal angle $+20^{\circ}$ ) and contr-ECCD (toroidal angle- $20^{\circ}$ ) and focused beams was

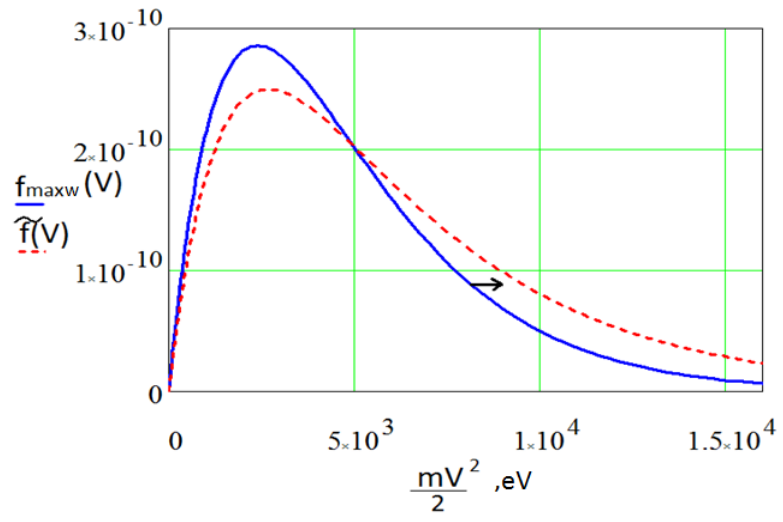

Fig. 1. Velocity distribution functions in terms of kinetic energy.

used in the series of shots (called later as co+contr). The new phenomenon has been found in some shots with $\mathrm{W}$ limiter and $\mathrm{Li}$-coating in the above mentioned regime.

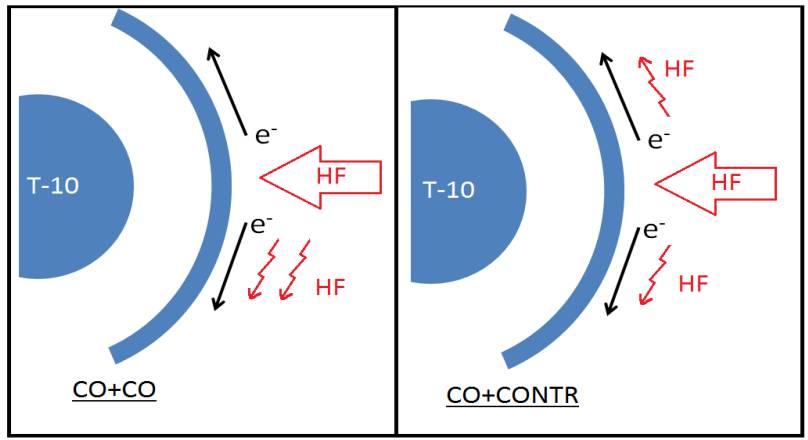

Fig. 2. Two types of HF injection. 
The spontaneous rise of the electron density nearly in the all plasma column occurs simultaneously with the rise of $\mathrm{Te}$ in the wide region $(0<\mathrm{r} / \mathrm{a}<0.8)$ at the steady-state phase of ECRH. The value of the energy confinement time abruptly rises by $\sim 15 \%$ remaining global L-H transition. Initially, non-local (global) confinement bifurcations were observed at 'fast' $\mathrm{L}-\mathrm{H}-\mathrm{L}$ transitions in some normal magnetic shear regimes of JET [1-4] and JT-60U [5]. The abrupt in time (ms timescale) and wide in space profiles of the electron and ion heat diffusivities variation $\delta \chi \mathrm{e}$, i (up to $90 \%$ of plasma volume or at $0.3<\mathrm{r} / \mathrm{a}<1)$ were found. The profile of $\delta \chi \mathrm{e}$ hardly penetrates into the RS region for the strong ITB. The $\delta \chi \mathrm{e}$ profile follows the position of $q$ min (minimum of q) and penetrates the RS region deeper for the weak ITB than for the strong one, suggesting 'global' edge-core connection penetrating through the position of $q_{\min }$ (up to shear value equal to $\approx-1.5$ ) on a ms timescale.

\section{T-10 tokamak and Gyrotron set-up}

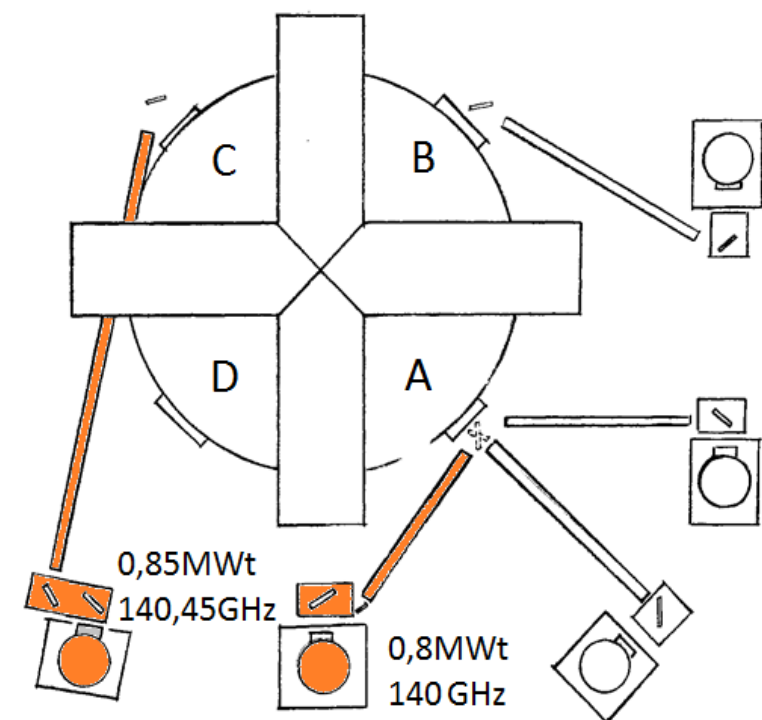

Fig. 3. Major radius $\mathrm{R}=1,5 \mathrm{~m}, \mathrm{~W}$-limiter $\mathrm{a}=0,3 \mathrm{~m}$. " $\mathrm{A}$ " and " $C$ " ports are supplied with scanning launchers with focusing beam. Beam waist $\rho=0,8 \mathrm{~cm}$.

Microwave radiation was injected at the stationary stage of the plasma discharge $(650 \mathrm{~ms}-850 \mathrm{~ms})$ by two gyrotrons simultaneously at an angle of 20 degrees in opposite directions on the second harmonic of ECR in $\mathrm{X}$ - mode. The zone of ECRH is located between the radius of the relativistic cut-off and the position of the cold resonance, which is about $7 \mathrm{~cm}$ for $\mathrm{T}-10$ gyrotron set-up.

With the toroidal magnetic field $\operatorname{Bt}(0)=2.42 \div 2.44 \mathrm{~T}$, the energy deposition has located in the central area of plasma column. Plasma current $\mathrm{Ip}=220 \mathrm{kA}, \quad<\mathrm{n}>=$ $2 \cdot 10^{13} \mathrm{~cm}^{-3}$.

\section{Non-local L-H transition at co+contr ECCD}

The timetraces of $\mathrm{Te}$ at the various radial positions are shown on the Fig. 4. Two gyrotrons with $\mathrm{P}_{\mathrm{ECRH}}=1.4$ MW are switched-on at $\mathrm{t}=700 \mathrm{~ms}$. The arrow shows the start of Te rise. The Fig.5 shows the behavior of the central line averaged density measured by interferometer. The electron density starts to rise in all plasma volume. Fig.6 represents evolution of the ne profiles before and $20 \mathrm{~ms}$ after transition (only 6 channels of the laser interferometer were in operation). In our opinion, the spontaneous rise of Te and $n_{e}$ represents $\mathrm{L}-\mathrm{H}$ transition. The rise of $(6+\mathrm{Li} / 2)$ appears at the same time and $\delta(\partial(b+\mathrm{Li} / 2) / \partial \mathrm{t})=0.25 \mathrm{MW}$ (the value of the energy content $\mathrm{W}$ was not measured in this shot). The value of the energy confinement time $\tau \mathrm{E}$ abruptly rises by $\sim 15 \%$. We restore the value of $\mathrm{W}$ (shot 68348 ) with the value $\mathrm{W}$ measured in similar shots without clear transition (e.g. 68355) by comparison of Te and ne profiles. Even after L-H transition the value of $\tau \mathrm{E}$ is equal to the predictions of ITER L-mode scaling (21 $\mathrm{ms})$. The rise of Te is not symmetric at LFS and HFS due to the increase of Shafranov shift.

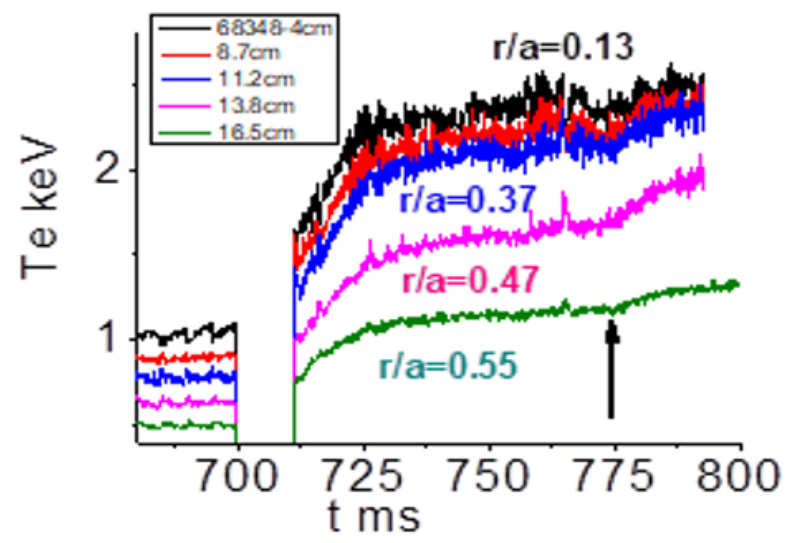

Fig. 4. Timetraces of $\mathrm{Te}$ in shot 68348 , the start of $\mathrm{Te}$ rise is shown by arrow.

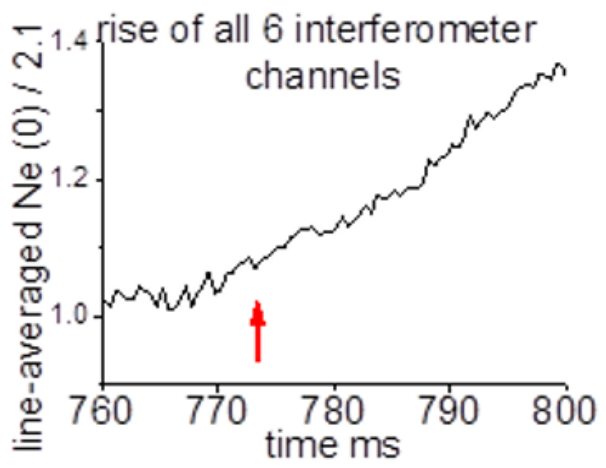

Fig.5.Timetraces of $\mathrm{Ne}(0) / 2.1$ line-averaged in shot 68348, the start of Ne rise is shown by arrow.

The Figure 7(a) shows the profiles of Te before and 20 ms after L-H transition. Besides the obvious asymmetry, internal transport barrier (ITB) forms at $\mathrm{r}=-15 \mathrm{~cm}$ and $\mathrm{r}=+18 \mathrm{~cm}$. The Figure 7 (b) represents the radial profile of $\delta(\partial \mathrm{Te} / \partial \mathrm{t})$ at the time of the transition multiplied by 10 
ms. Asymmetry at LFS and HFS is observed also. Since the gradient of Te nearly not varies at the time of $\mathrm{L}-\mathrm{H}$ transition, the simple expression (2) allows us to calculate the jump of the electron heat flux $\delta \Gamma$ e from the simple equation ( $\mathrm{S}$ is the enclosed surface, $<>$ means averaging in a few ms time interval after transition) [1$6]$.

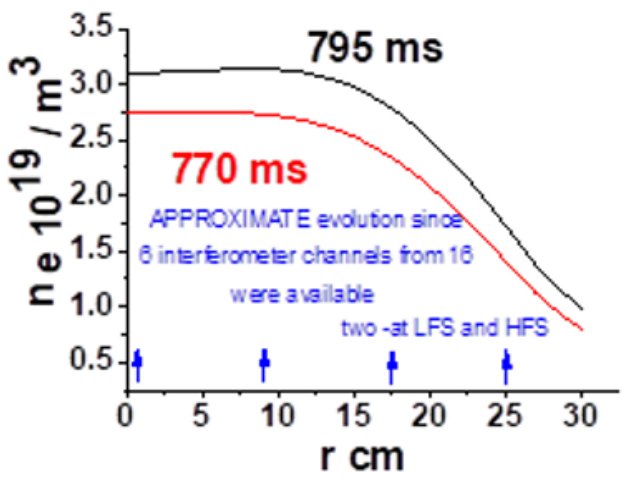

Fig. 6. Density profiles before and $20 \mathrm{~ms}$ after L-H transition.
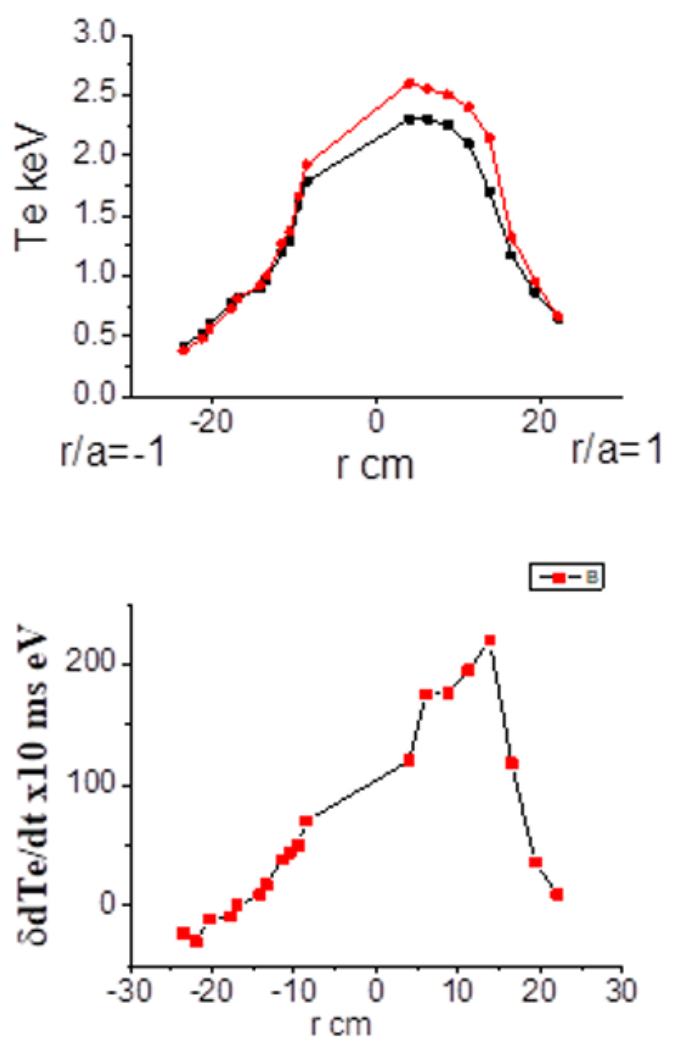

Fig.7. (a) Profiles of Te before and $20 \mathrm{~ms}$ after $\mathrm{L}-\mathrm{H}$ transition (b) The radial profile of $\delta(\partial \mathrm{Te} / \partial \mathrm{t}) \times 10 \mathrm{~ms}$ at the $\mathrm{L}-\mathrm{H}$ transition.

$$
\begin{aligned}
& <\delta \Gamma e(r)>S(r)=-n e<\delta \chi e>\nabla(T e) S(r)= \\
& -3 / 2 \int_{0}^{V(r)} \delta<(\partial(n e T e) / \partial t)>d V
\end{aligned}
$$

The profile of $\delta \Gamma_{\mathrm{e}}(\mathrm{r})$ is shown on the Figure 8. The rise of ne is important in this particular case since $\delta(\partial \mathrm{Te} / \partial \mathrm{t}) / \mathrm{Te}<\delta(\partial \mathrm{ne} / \partial \mathrm{t}) /$ ne in the outer half of plasma column.

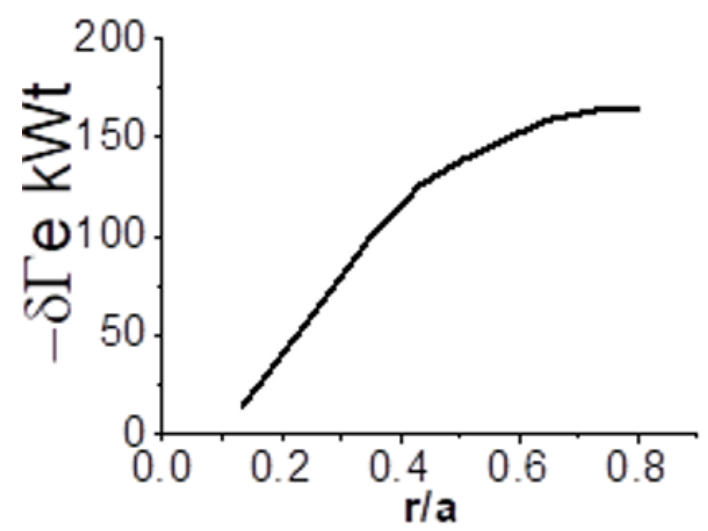

Fig 8 Radial profile of $\delta \Gamma_{\mathrm{e}}(\mathrm{r})$ at the $\mathrm{L}-\mathrm{H}$ transition

The profile of $\delta \Gamma_{\mathrm{e}}(\mathrm{r})$ shown on the Figure 8 is very wide in space reminding that of at non local L-H transitions in JET and JT-60U [1-6], see detail in the Introduction above. The ion energy rises also since $\mathrm{Ti}$ (not well measured in shot 64348) at least did not drop after transition and $n_{\mathrm{e}}$ raised. The $15 \%$ rise of $\tau_{\mathrm{E}}$ at the time of L-H transition shown above does not contradicts with the joint reduction of electron and ion heat fluxes. The electron density flux reduces in all plasma volume since the density rises in all plasma column. The level of the plasma radiation measured at the various radial positions with AUX bolometers (sensitive to the radiation of the tungsten [7]) drops also. The density of the tungsten ions reduces. The accumulation of the light impurities is absent since SXR data shows proper rise (linear to small values of $\delta$ Te and square to the rise of ne).

\section{Co+co and co+contr injection}

Shots in which L-H transition was not observed were selected for a detailed comparison of the plasma parameters for $\mathrm{co}+\mathrm{co}$ and co+contr $\mathrm{HF}$ injection. Experiments, in particular, showed that the increment of the average electron concentration $n_{e}(0)$ is higher in the case of co+contr injection in comparison with co+co, with the same "pump-out" effect, arising after gyrotrons start. Density feedback control was operated identically. The average value of electron density continued to grow during of co+contr injection and reaches a value greater than the initial value (see Fig.9). Also, the change of the shape and period of sawtooth oscillations was observed. 
For the purity of the experiment, a series of pulses with an additional gas-puffing was carried out in the case of co + co injection in order to obtain the evolution of a density identical to co+contr case (see Fig.10).

As a result, with the same time evolution of electron density in the central region of the plasma, which varied from $\mathrm{n}_{\mathrm{e}}(0)=3.0 \cdot 10^{13} / \mathrm{cm}^{3}$ to $\mathrm{n}_{\mathrm{e}}(0)=3.8 \cdot 10^{13} / \mathrm{cm}^{3}$ during the time interval with the linear rise of the density (see Fig. 10), the central electron temperature measured by ECE was $\mathrm{Te}(\mathrm{r} / \mathrm{a}=0.15)=2.4 \mathrm{keV}$ at the quasi-stationary stage for both HF injection methods (see Fig.11).Thus, the observed value of $n_{e} \times T_{e}$ in the central region of the plasma column was the same.

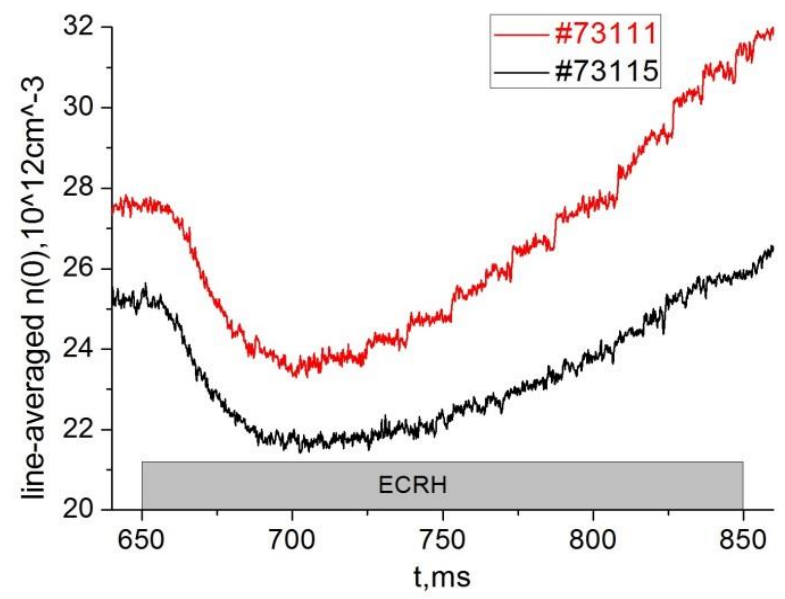

Fig. 9. Line-averaged $n_{e}(0)$ time evolution, shot \#73111co+contr ECCD, shot \#73115- co+co ECCD.

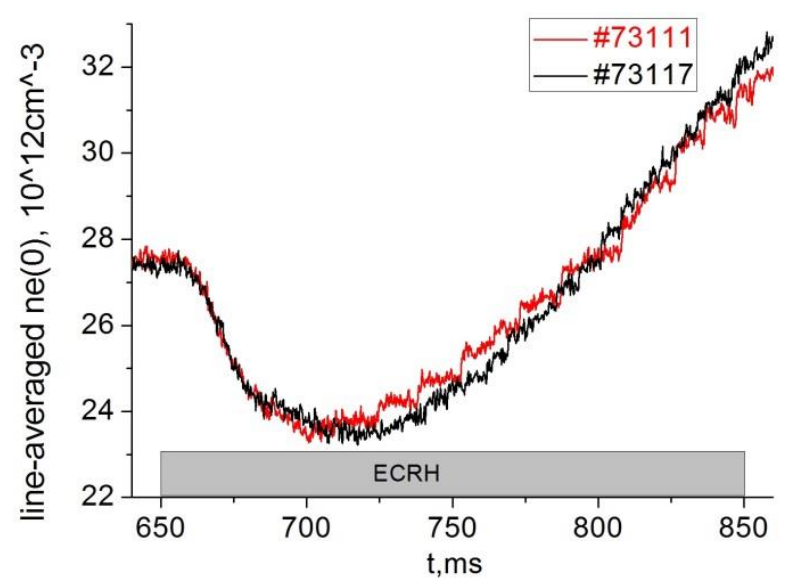

Fig.10. Line-averaged $n_{e}(0)$ time evolution. (\#73111co+contr), (\#73117- co+co with additional gas-puffing).

Spectrum analysis was carried out in the range of 8-14 $\mathrm{keV}$ because of the poor statistics at energies above 14$16 \mathrm{keV}$.

However, the measurements of X-ray spectra (PHA data) showed a significant difference. (see Fig.12).

The approximated "temperatures", which were made after averaging of the PHA statistics, have values of
$3.1 \mathrm{keV}$ for co+co injection and $3.56 \mathrm{keV}$ for co+contr injection (see Fig.13).

If we take into account that the measurements on the second harmonic ECE for collision plasma are very weakly dependent on the longitudinal energy of electrons, and X-ray spectrum is practically isotropic up to $100 \mathrm{keV}$, we can assume that a significant part of the electron energy is contained in the longitudinal component. Moreover, in the case of co+contr injection longitudinal energy of the electrons is greater than for co+co injection. If we suppose, for example, that the mean value of kinetic energy of the longitudinal component is close to the transverse value, the energy content in the resonance zone increases by $\approx 30 \%$.

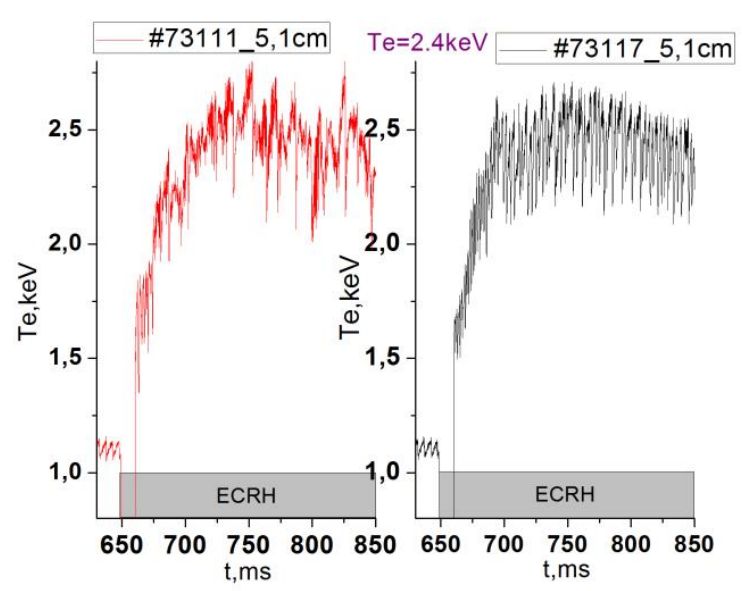

Fig.11. Time evolution of $\mathrm{Te} \mathrm{ECE}_{\mathrm{E}}(5 \mathrm{~cm})$ in comparable shots \#73111 (co+contr) and \#73117 (co+co).

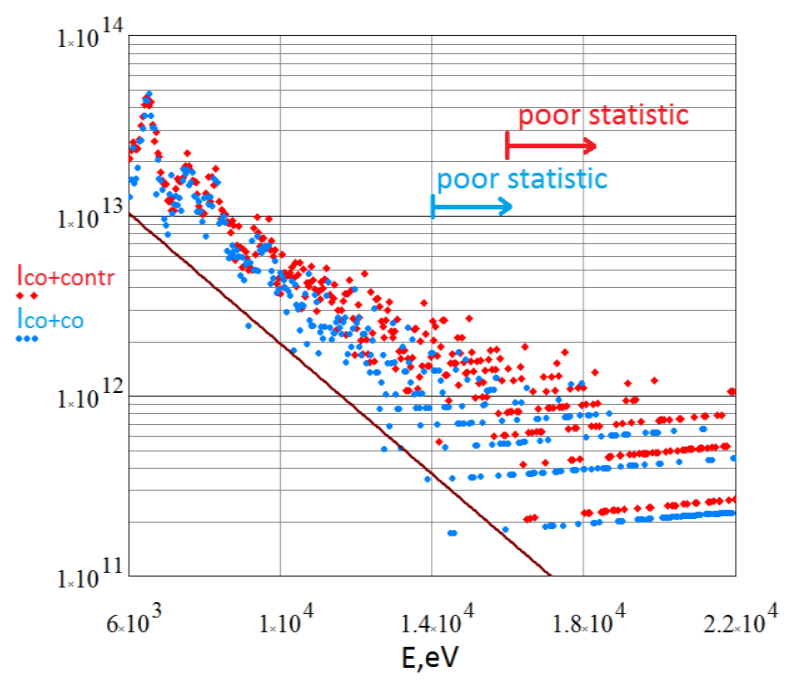

Fig. 12. • X-ray spectrum (\#73111- co+contr, red circles), $X$ ray spectrum (\#73117- co+co, blue circles).

\section{Conclusion}

The new phenomenon has been found in some shots with W-limiter (and Li-coating) in the plasmas with two EC- 
beams with power injected in opposite directions under approximately equals toroidal angles and EC-power 1.5 MW. The spontaneous rise of the electron density in the all plasma column occurs simultaneously with the rise of Te in the wide region $(0<\mathrm{r} / \mathrm{a}<0.8)$. We treat it as "nonlocal or global L-H transition" found earlier at JET [1-4] and in various regimes of JT-60U [5-6]. The value of Dbeta falls, the positive value of $\mathrm{dW} / \mathrm{dt}$ appears and the value of the energy confinement time $\tau \mathrm{E}$ abruptly rises by $\sim 15 \%$. The absolute value of the electron heat and density fluxes reduces abruptly in the whole plasma column. The accumulation of tungsten and light impurities is absent. By our up-to-date knowledge, the non-local L-H transitions never have been described in limiter tokamaks with circular cross-section.

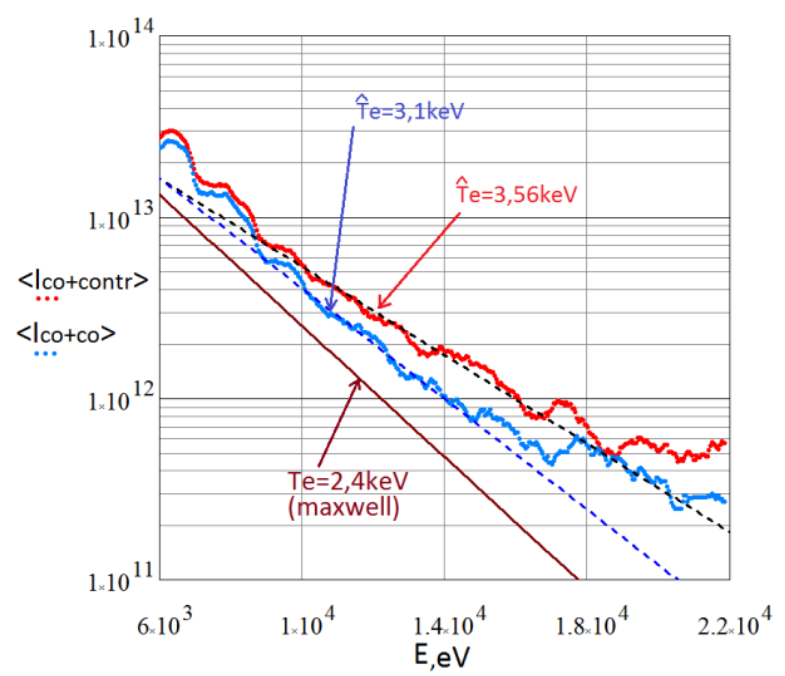

Fig.13. Approximated values of "electron temperature". Brown line corresponds to the temperature for Maxwellian distribution.

Preliminary analysis of X-ray spectra (PHA) shows, that in the case of co+contr injection the central area of plasma, where HF is deposited, becomes significantly non-Maxwellian.

We assume, that this phenomenon related to higher mean value of longitudinal energy of electrons in the resonance zone.

$\mathrm{Co}+$ contr injection provide more energy content in the central area of plasma column in comparison with co + co injection.

The energy value can be higher in relation to the energy in the Maxwell distribution up to 20\% -30\%.

Resonance zone can be expanded by application of additional HF injections with slightly changed toroidal and poloidal angles and also frequency.

This heating method, together with the possible L-H transition can yield even greater energy content of the plasma.

We indebted to the members of $\mathrm{T}-10$ team participated in the experiments described in the present paper.

\section{References}

1. S.V. Neudatchin, J.G. Cordey and D.J.Muir 20th EPS Conf. on Control. Fus. and Plasma Phys. (Lisboa,) vol. I (Geneva : EPS), p 83 (1993)

2. S.V. Neudatchin, J.G. Cordey and D.J.Muir The time behaviour of the electron conductivity during L-H and H-L transitions in JET JET Rep. JET-P (93)-58 (Jet Joint Undertaking Abingdon Oxforshire UK) (1993)

3. J.G.Cordey, D.J. Muir, S.V. Neudatchin et al Plasma Phys. Control. Fusion 36 A267 (1994)

4. J.G. Cordey, D.J. Muir, S.V. Neudatchin et al, Nucl. Fusion 35505 (1995)

5. S.V. Neudatchin, T. Takizuka, H. Shirai et al., Japan J. Appl. Phys. 353595 (1996)

6. S. V. Neudatchin, T. Takizuka, H. Shirai et al., Plasma Phys. Control. Fusion 44 A383-389 (2002)

7. V.A. Vershkov et al Nucl Fusion 2017347 